\title{
Hypothalamic Regulatory Hormones
}

\author{
Abstracts from the Joint Meeting of the Swedish Society for Endocrinology \\ and the Swedish Society for Gynaecology in Uppsala, 20th September, 1975.
}

The Hypothalamus as an Endocrine Organ. By G M. Besser, The Medical Professorial Unit, St. Bartholomew's Hospital, London, EClA 7BE, England.

The hypothalamus is associated with the synthesis of two distinct groups of hormones. The longest recognised group-vasopressin, oxytocin and neurophysin-is made in the neurones of the supraoptic and paraventricular nuclei and pass down the neuronal axons of these cells ('neurosecretion') through the pituitary stalk to be stored in their terminal regions in granulated swellings in the posterior pituitary. The other more recently recognized group-the hypothalamic regulatory hormones-is also made in the hypothalamic nuclei and neurosecreted down the axons to be stored in the region of the median eminence. When released these substances pass down the portal capillary system of the stalk to act on the anterior pituitary cells to cause them either to synthesise and release their hormones, or to inhibit the release. Release of the stored hypothalamic hormones in both groups occurs under the influence of neurotransmitters released in response to neurogenic stimuli from above or as the result of feedback influences of circulating hormones. The process of signal transfer: hypothalamus-median eminence-anterior pituitary-target gland-systemic tissues and behavioural responses, results in a great amplification of the initiating signal and allows for sensitive feedback control at all levels.

Six hypothalamic regulatory hormones are clearly recognized: releasing hormones for thyrotrophin (TRH), the gonadotrophins (Gn-RH), ACTH, GH, prolactin; release-inhibiting hormones for prolactin (PRIH) and growth hormone (GH-RIH). The structures for TRH (a tripeptide), Gn-RH (a decapeptide) and GH-RIH (a cyclized tetradecapeptide) are established and many believe that the most important PRIH is dopamine. The structures of the others are unknown. The actions of these hormones are not pure. In normal subjects TRH releases TSH and prolactin and even a little FSH in males, GnRH releases both gonadotrophins but in adults more $\mathrm{LH}$ than $\mathrm{FSH}$, and $\mathrm{GH}-\mathrm{RIH}$ inhibits release of GH, TSH, insulin, glucagon, gastrin, vasoactive investinal polypeptide and renin and even inhibits platelet agglutination. The physiological significance of the diversity of actions is not clear but these substances may be neurotransmitters present both in the nervous system and elsewhere (TRH and GH-RIH are found intracellularly in the gut wall) but which have a specialized local function in the hypothalamic pituitary axis. The clinical potential of their compounds is still being explored. TRH is established as a diagnostic test in primary thyroid disease since in hyperthyroidism the TSH response to TRH is suppressed, and in hypothyroidism it is excessive. Administration of $\mathrm{Gn}-\mathrm{RH}$ will demonstrate the reserve capacity for $\mathrm{LH}$ and FSH secretion and on long term administration has been shown to successfully treat the infertility of some hypogonadotrophic patients. GH-RIH does inhibit secretion of excess $\mathrm{GH}$ in acromegaly, glucagon and $\mathrm{GH}$ in diabetes mellitus, and the excess hormonal secretion of some malignant tumours. However, in view of its platelet toxicity, its final role in clinical practice is not clear. The long-acting dopaminergic agent, bromocriptine, could be considered to be an analogue of PRIH. It has been used to suppress prolactin secretion in male and female patients with hyperprolactinaemia and successfully reverses their infertility and hypogonadism. Dopamine as well as bromocriptine also lowers GH in acromegaly, but not in normal subjects, and is used to treat acromegaly.

\section{Review References}

Besser, G. M.: Br Med J 3:560-544 and 613-615, 1974. Besser, G. M.: J Clin Pathol 27: 173-184, 1974.

Thorner, M. O. et al.: Br Med J 1:299-303, 1975. 
Localisation of Hypothalamic Hormones (LH-RH, TRH and Somatostatin) and Neurotransmitters (Monoamines and Substance P). By T. Hökfelt, K. Fuxe, O. Johansson, M. Goldstein, S. Efendic, R. Luft, G. Nilsson, B. Pernow, A. Arimura and S. Jeffcoate, Departments of Histology and Pharmacology, Karolinska Institute and Departments of Clinical Physiology and Endocrinology \& Metabolism, Karolinska Hospital, Stockholm, Sweden.

With the indirect immunofluorescence technique some hypothalamic hormones-luteinizing hormone-releasing hormone (LH-RH), tyreotrophin releasing hormone (TRH) and somatostatin (growth hormone release-inhibiting hormone)-has been studied in the peripheral and central nervous system. Furthermore, the distribution of monoamine neurons was studied in the same way with antibodies to the four catecholamine (A) synthesizing enzymes and, finally, the occurrence of Substance $\mathbf{P}$ (SP), a putative excitatory peptidergic neurotransmitter was studied with the same technique. In this way it is hoped to obtain a crude estimate of the morphological relationships between, on the one hand, neurons containing different types of transmitters and, on the other hand, the hormone producing systems.

The hypothalamic hormones are all found in the external layer of the median eminence but with a different distribution: LH-RH is located mainly in the lateral external layer, TRH mainly in the medial external layer and somatostatin in the entire external layer but in addition, and mainly, in the medial parts including the internal layer. Whereas many TRH and somatostatin positive nerve endings are found in the stalk, LH-RH axons seem to enter this structure only to a small extent. LH-RH positive cell bodies are found in the most frontal suprachiasmatic region and somatostatin positive cell bodies in the periventricular area.

Extensive intrahypothalamic and extrahypothalamic TRH, somatostatin and to a lesser extent LH-RH positive nerve terminal networks not related to blood vessels are also observed.

These findings will be discussed in relation to the different neurotransmitters and to functional aspects.
The Gonadotropin Response after Administration of LH/FSH-releasing Hormone (LRH) during Early Pregnancy. By Sten Jeppsson, Gunnar Rannevik and Jan Thorell, Department of Obstetrics and Gynaecology, University of Lund, General Hospital, Malmö, Sweden.

The basal levels of FSH in plasma are low during pregnancy. Furthermore the FSH-response after administration of LRH seems to be inhibited.

In an attempt to show the decreasing responsiveness of the pituitary to LRH during early pregnancy we carried out tests with $25 \mu \mathrm{g}$ LRH i.v. in 4 healthy women at the time of conception and then weekly during the first two months of pregnancy. The investigation showed that the basal levels of FSH decrease successively during the first few weeks of pregnancy. During the same period of time the pituitary responsiveness to LRH decreases and is completely inhibited in all women within 6-7 weeks after conception. In two of the patients the response was inhibited already $3-4$ weeks after conception.

Earlier studies have shown that this inhibition persists throughout pregnancy and up to 8-10 days after delivery at full term.

FSH and LH Response to Gonadotrophine-releasing Hormone in Patients with Hypogonadism of Hypothalamic or Hypophyseal Origin. By K. Brismar, P. Eneroth and K. Hall, Department of Endocrinology, Karolinska Hospital, 10401 Stockholm, Sweden.

The effects of an i.v. injection of synthetic LH-RH on FSH and LH levels in serum were studied in 31 patients with secondary hypogonadism as well as in control subjects

Material and methods. A single dose of $100 \mu \mathrm{g}$ synthetic LH-RH (Hoechst) was given as an i.v. injection to 52 subjects aged 15 to 40 years. The following two groups of subjects were compared: (1) A control group comprising 21 subjects $(9$ males and 12 females) without any clinical signs of hypogonadism. Ten of these subjects had verified hypophyseal tumors. (2) A group of 31 subjects (20 male, 11 female) with secondary hypogonadism of various origin. Twelve of these subjects had hypo- 
thalamic disorders such as Kallman's syndrome and hypothalamic tumors and 12 others had hypophyseal tumors. Seven subjects had hypogonadism and pituitary dwarfism of unknown origin. Serum samples were drawn at $-10,0,+20,+30,+60$ and +120 minutes after the injection. FSH and $\mathrm{LH}$ were determined by radioimmunoassay using kits from Serono, Italy. A solid phase second antibody (DASP, Organon) was used. Values obtained were related to the second IRP-hMG standard.

Results. The increase of FSH and LH levels in serum after LH-RH was significant in the group of controls. There was no correlation between the maximum response obtained for FSH and that for LH. The serum levels of gonadotrophins during basal condition as well as after LH-RH were significantly lower in the groups of patients with hypogonadism that those in the control group. However, there was an overlapping of $\mathrm{FSH}$ and $\mathrm{LH}$ levels between the subjects with hypogonadism and the control subjects both during basal condition and after LH-RH. The LH-RH test improved the separation somewhat, but this test could not distinguish between patients with hypogonadism of hypothalamic or hypophyseal origin.

TRH- and LH-RH-tests in the Evaluation of Patients with Tumours of the Sellar Region. By P. O. Lundberg and L. Wide, Department of Neurology and Department of Clinical Chemistry, University Hospital, Uppsala, Sweden.

Intravenous TRH $(200 \mu \mathrm{g})$ and LH-RH $(100 \mu \mathrm{g})$ was given to study the effect on blood TSH and LH respectively in about 150 cases with tumours of the sellar region. The increase of blood hormones was compared with the response in certain control groups (healthy volunteers of different sex and age, women with "functional" amenorrhea).

An absent or subnormal response to LH-RH was seen in almost all of the cases with craniopharyngiomas or chromophobe adenomas with both intraand suprasellar extension, but in none of the cases with non-functioning intrasellar tumours or in the meningioma or glioma cases. The results of the TRH-tests were similar. Thus, these tests cannot be used to exclude a tumour of the sellar region but an absent or subnormal response usually indicates a big tumour destructing most of the pituitary.
In 45 cases the tests were repeated after a period of time (up to 3 years) to study the effect of surgery and X-ray-therapy or a recurrence of the tumour. These studies are still in progress but the following preliminary observations have been made. Patients with normal response before surgery have not been found to regain a normal $\mathrm{TSH}$ or $\mathrm{LH}$ secreting capacity after surgery. A number of cases with small tumours in the suprasellar region-not extending into the pituitary fossa-had completely normal responses to both tests before as well as after surgery.

\section{A Depot Preparation of Luteinizing Hormone-releas-} ing Hormone. By T. Bergh, S. J. Nillius, J. Sandow and L. Wide, Departments of Obstetrics and Gynaecology and Clinical Chemistry, University Hospital, Uppsala, Sweden and Department of Pharmacology-Endocrinology, Farbwerke Hoechst AG, Frankfurt (M.), Germany.

The present therapeutic regimens of luteinizing hormone-releasing hormone ( $\mathrm{LH}-\mathrm{RH})$ are not very practical. LH-RH has a short biological half-time and multiple injections of LH-RH have to be given each day over long periods to stimulate gonadal function. For therapeutic purposes, it would be advantageous to have a long-acting preparation. Superactive analogues of LH-RH are presently tested in the human. However, before such analogues can be used for long-term therapy they have to be carefully studied with regard to toxicity, immunogenicity etc. as they are not naturally occurring substances. Synthetic LH-RH, however, has been used extensively over the past four years in numerous clinical investigations without showing any side effects. It might therefore be preferable to try to potentiate the action of the "natural hormone" by the use of depot carriers for LH-RH.

We have made a pharmacological and clinical evaluation of a depot preparation of LH-RH. This preparation, LH-RH Depot, was developed by using a derivative of gelatine (Haemaccel ${ }^{\circledR}$, Hoechst) as a depot carrier for LH-RH. In male rats, the depot preparation with $10 \mu \mathrm{g}$ of $\mathrm{LH}-\mathrm{RH}$ increased the total LH release during four hours by $256 \%$ and the total FSH release by $238 \%$ in comparison with the corresponding dose in saline. In adult female rats or immature female rats pretreated with preg- 
nant mare serum gonadotropin, the ovulation rate was significantly higher after treatment with $\mathrm{LH}-$ RH Depot than with LH-RH in saline.

LH-RH Depot was then tested in four women with secondary amenorrhoea. One hundred $\mu \mathrm{g}$ of LH-RH in the depot preparation was given intramuscularly. The effects on the serum levels of FSH and LH were compared with those obtained with the same dose of LH-RH in saline. Immunoreactive FSH and $\mathrm{LH}$ in serum were assayed by a radioimmunosorbent technique. LH-RH in saline resulted in a rapid release of $\mathrm{LH}$ with the peak response $30 \mathrm{~min}$ after the injection. The mean $\mathrm{LH}$ level remained elevated above the basal level for at least 8 hours. LH-RH Depot administration resulted in a slower LH release with the peak response 3 hours after the injection. The mean LH levels obtained 4-7 hours after the LH-RH Depot injection were significantly higher than those seen with the same dose of LH-RH in saline. There was no difference in the mean FSH levels obtained during the first two hours after administration of the two preparations. However, during the remainder of the study period (3-8 hours) the mean FSH levels obtained with LH-RH Depot were significantly higher than those obtained with LH-RH in saline.

Thus, administration of LH-RH with a depot carrier resulted in an augmented and prolonged effect on the release of FSH and LH in rats and amenorrhoeic women, in comparison with LH-RH in saline. Further studies have to be made to decide if depot preparations of LH-RH are useful for clinical therapeutic application.

\section{Therapeutic Use of Luteinizing Hormone-releasing Hormone in the Human Female. By Sven Johan Nillius and Leif Wide, Departments of Obstetrics \& Gynaecology and Clinical Chemistry, University Hospital, Uppsala, Sweden.}

The exciting possibility of using synthetic luteinizing hormone-releasing hormone ( $\mathrm{LH}-\mathrm{RH})$ instead of human gonadotropins for induction of follicular maturation and ovulation was studied in 13 amenorrhoeic women who had no evidence of endogenous ovarian activity before the treatment.

L.H-RH, $500 \mu \mathrm{g}$ every eight hours, was administered intramuscularly or subcutaneously dur- ing 16 treatment cycles. Follicular maturation, as judged by increased oestrogen levels in blood, was produced after 9-23 days of treatment in all but two cycles. Ovulation, as judged by increased progesterone levels in blood, was also induced in seven patients who were treated with LH-RH alone. However, the progesterone values during the luteal phases in four of these induced cycles were rather low, suggesting insufficient corpus luteum function. LH-RH was therefore combined with human chorionic gonadotropin (HCG) during seven treatment cycles. Six of these cycles were ovulatory with normal luteal phase progesterone values. Three involuntarily sterile amenorrhoeic women were treated with LH-RH combined with HCG. One of them conceived and by that she proved that ovulation and normal corpus luteum function occurred during the LH-RH-HCG treatment.

Thus, it seems possible to replace human gonadotropins with LH-RH for induction of follicular maturation and ovulation at least in some women with amenorrhoea. Replacement of HMG by LH-RH would probably have many advantages. Hyperstimulation of the ovaries might be easier to prevent during $\mathrm{LH}-\mathrm{RH}$ treatment. It is known that gonadal steroids can modulate the pituitary responsiveness to LH-RH. The intact feedback system between the pituitary and the ovaries which is present in patients treated with LH-RH, represents an internal control mechanism, which may automatically prevent excessive FSH stimulation of the ovaries during prolonged treatments with LH-RH. However, many more clinical studies must be performed before we can define the proper place for this gonadotropin-releasing hypothalamic hormone in the therapy of anovulatory infertility. 